\title{
Analysis and Experiments on Strengthening the Platform Jacket by the Grouted Clamps
}

\author{
Zhuo WANG ${ }^{1}$, Bo ZHANG ${ }^{1, a}$, Tao WANG ${ }^{2}$ \\ ${ }^{1}$ Harbin Engineering University, College of Mechanical and Electrical Engineering, 150001 Harbin, \\ China \\ ${ }^{2}$ Hebei University of Technology, School of MechanicalEngineering, 300401 Tianjin, China \\ azhangbo_heu@hrbeu.edu.cn
}

Keywords: Damaged pipe, Finite element analysis, Experiments in mechanics, Grouted clamps, Offshore platform;

\begin{abstract}
The grouted clamps will strengthen the offshore platform jackets which have fragile nodes and the damaged structure underwater. Theoretical analysis of buckling on the jacket was done with buckling theory under uniform axial compression and the method of finite element analysis under the condition of pipeline before and after the installation used by grouted clamp in order to ensure the reliability and safety of the offshore platforms. Experiments of grouted clamps strengthening the damaged pipe had been made and verified its reinforcement effect. This method of combining theory with experiments has sufficiently shown that the strengthening device of grouted clamp is a simple and feasible reinforcement method; it can solve the difficulties in offshore platforms nowadays by local strengthening technology on contact node of pipe and underwater steel bars.
\end{abstract}

\section{Introduction}

The structure of an offshore platform becomes more and more completed in its form and function in the development of technology of developing oil and gas resources. And the quality of an offshore platform structure gets more attention with the increased requirements of environmental protection and production safety [1]. The structure of an offshore platform has lots of features, including a huge frame, complicated structure, expensive cost, complex and harsh environment etc [2]. In the extremely complex and harsh environmental conditions, the risk factors resulting in damage to offshore platform structure include the foundation erosion, falling objects, ship collisions, increasing seawater corrosion, adhesion of marine organisms, materials aging, flaws of manufacturing and design and misuse in the installation process[3-6]. Local damages caused by them reduce the safety and reliability of the offshore platform structure and affect the normal development of oil and gas resources [7]. Offshore platform structure is the key equipment to develop oil and gas resources, so improving its safety and reliability is essential [8-10]. In this paper, mechanical analysis and experimental research of reinforcement of grout clamp will be done to verify the effect of strengthening and reinforce on the offshore platform.

\section{Buckling Theory Analysis of the Reinforcement of Grout Clamp}

\section{Buckling Theory Analyses of Reinforcement of Grout Clamp under Uniform Axial Compression}

It is assumed that the pipeline is bored by the uniform axial compression and the buckling deformation is shown in Figure 1. 


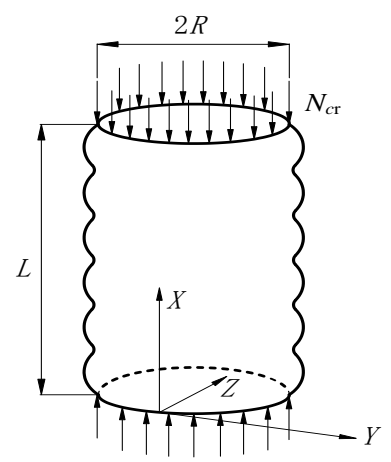

Fig. 1. Buckling under uniform axial compression

The critical buckling value of the pipeline under uniform axial compression can be solved by the Karman-Donnel nonlinear buckling equations. Karman-Donnell equations are introduced in 1934 by Donnell with the assumptions about the simplifying structure of the shell plate in Karman large deflection theory and get the balance equation of simplified thin shell, so it is called Karman-Donnel large deflection balance equation [11]:

$$
D \nabla^{4} w-\frac{1}{R} \frac{\partial^{2} \varphi}{\partial x^{2}}=\Lambda(w, \varphi)
$$

According to equation (1), the critical pressure of the pipeline under uniform axial compression was derived as follows

$$
\sigma_{c r}=\frac{2 E}{\sqrt{3\left(1-\mu^{2}\right)}(D / t)}
$$

Where: $t$ is the pipe wall thickness, $\mathrm{mm} ; D$ is the pipe diameter, $\mathrm{mm} ; \mu$ is the Poisson's ratio; $E$ is the modulus of elasticity, MPa.

The pipeline installed grouted clamp is equivalent to three homogeneous layer with composite layer equivalent theory, and the formula of pipeline installed grouted clamp was derived by the solving equation of pipeline under uniform axial compression as follows [12]:

$$
\sigma_{c r}=\frac{2}{\sqrt{3} D_{0}} \sum \frac{E t}{\sqrt{\left(1-\mu^{2}\right)}}
$$

Where: $D_{0}$ is the average diameter, $\mathrm{mm}$.

A series of data was selected to describe the reinforcement effect of grouted clamp for pipeline and calculated by equation (2) and (3).At last, the critical pressure was contrasted by date fitting before and after installing the clamp.

The data in Table 1 are the critical pressure of the pipeline under uniform axial compression before and after installing the clamp as showed.

Table 1. Critical pressure before and after installing clamp

\begin{tabular}{|c|c|c|c|}
\hline $\boldsymbol{D}_{\mathbf{1}} / \boldsymbol{t}_{\mathbf{1}}$ & Without clamp $\boldsymbol{\sigma}_{\boldsymbol{c}}(\mathbf{M P a})$ & With clamp $\boldsymbol{\sigma}_{\boldsymbol{c}}(\mathbf{M P a})$ & Difference(MPa) \\
\hline 20 & 12467.700 & 17975.000 & 5507.300 \\
\hline 22 & 11220.930 & 16890.000 & 5669.070 \\
\hline 25 & 9974.160 & 15805.000 & 5830.840 \\
\hline 29 & 8727.390 & 14720.000 & 5992.610 \\
\hline 33 & 7480.620 & 13635.000 & 6154.380 \\
\hline 40 & 6233.850 & 12550.000 & 6316.150 \\
\hline 50 & 4987.080 & 11465.000 & 6477.920 \\
\hline 67 & 3740.310 & 10380.000 & 6639.690 \\
\hline 100 & 2493.540 & 9295.000 & 6801.460 \\
\hline 200 & 1246.770 & 8210.000 & 6963.230 \\
\hline
\end{tabular}


According to Table 1, the relationship between buckling critical pressure of the pipeline under uniform axial compression before and after installing grouted clamp and diameter to thickness ratio as showed in Figure 2.

As can be seen from the figure, the critical buckling pressure of pipe after installing clamps in comparison with the pipe before installing clamp significantly increased. The critical pressure of pipe increases under uniform axial compression which indicates that the ability to resist external damage has increased. Thus the grouted clamp not only plays a role in repair but also plays a role in strengthening. When a pressure makes the pipeline damage, it cannot make the pipeline which is installed grouted clamp damage. It also can be seen from the figure, the greater the diameter to thickness ratio, the greater the critical pressure difference. With the increasing of diameter to thickness ratio, the critical pressure difference gradually into a horizontal range.

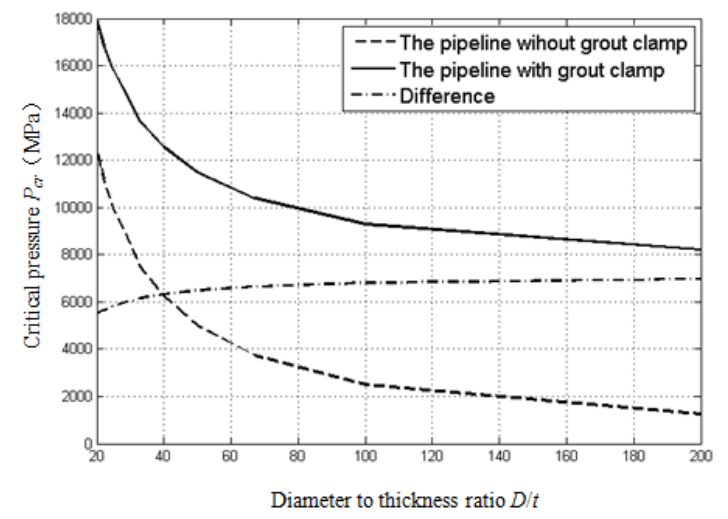

Fig. 2. The buckling critical pressure and the diameter to thickness ratio before and after installing grout clamp

\section{Buckling Analyses of the Pipeline Based on ANSYS under Uniform Axial Compression}

Finite element buckling analysis of pipeline under uniform axial compression can be further validated reinforcement effect for grouted clamp to pipeline. Importing the three-dimensional model into buckling analysis module, adding material properties, meshing and adding constraints and loads were done, then solved according to the ten models. Ten modal buckling analyses were done for pipeline before and after installing grouted clamp and the load multipliers of each model were solved to get the buckling pressure. When pressure exceeds the solved buckling pressure, the pipe will be instability. Solved results are shown in Table 2.

Table 2. Load multipliers of each modal

\begin{tabular}{|c|c|c|c|c|c|}
\hline & Modal 1 & Modal 2 & Modal 3 & Modal 4 & Modal 5 \\
\hline Pipe & $9.452 \mathrm{e}-7$ & $3.472 \mathrm{e}-6$ & 5.739 & 5.745 & 20.219 \\
\hline Clamp & $8.312 \mathrm{e}-6$ & $9.857 \mathrm{e}-6$ & 3.893 & 27.243 & 27.244 \\
\hline & Modal 6 & Modal 7 & Modal 8 & Modal 9 & Modal 10 \\
\hline Pipe & 20.221 & 21.504 & 21.507 & 24.445 & 24.446 \\
\hline Clamp & 27.247 & 27.248 & 27.384 & 27.385 & 27.389 \\
\hline
\end{tabular}

The axial load applied is $100 \mathrm{MPa}$ in the calculation process and the load multipliers in table 3 multiplied by 100 to get buckling load pressure. The buckling pressure of each model before and after installing grouted clamp and difference were described as showed in Figure 3. 


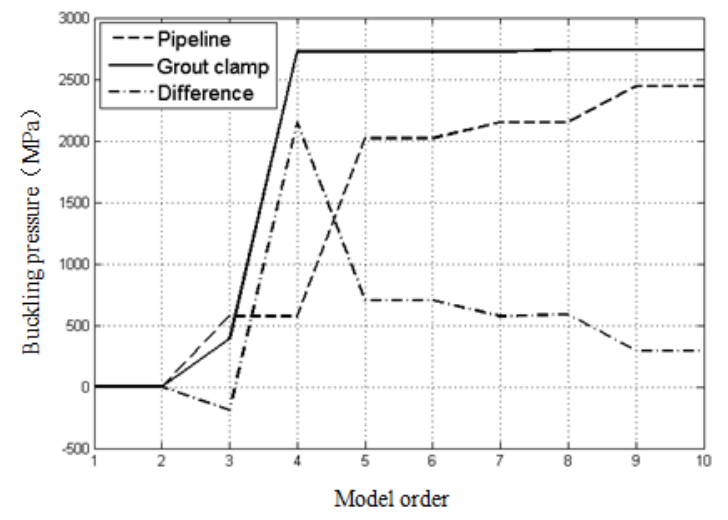

Fig. 3. The contrast between buckling pressure before and after installing grout clamp

As can be seen from the figure, the buckling pressure of pipeline installed with grouted clamp is larger than the pipeline under uniform axial compression. And the greater of the modal, the smaller of the difference. But it is enough to prove repair and reinforcement effect for grouted clamp to damage the pipeline.

Buckling analysis of grouted clamp has been demonstrated from the aspect of solving data. It also can be got from the finite element analysis cloud to verify the role of reinforcement of grouted clamp to pipeline. Below are given the order 1, 3,5,7,9 pipes buckling deformation cloud before and after installing grouted clamp as showed in Figure 4 and Figure 5.

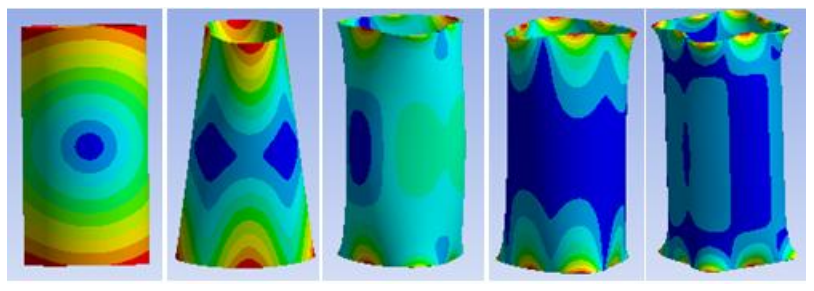

Fig. 4. deformation of pipeline before installing clamp

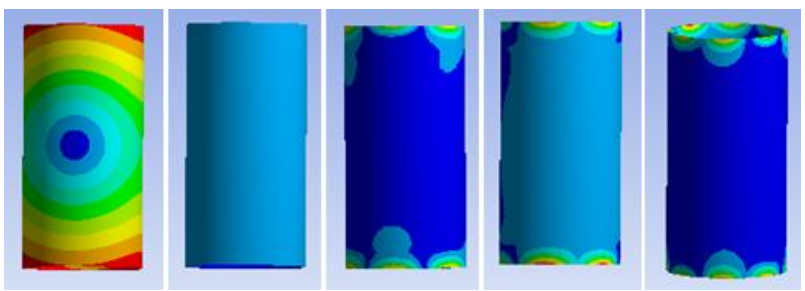

Fig. 5. deformation of pipeline after installing clamp

As can be seen from the figure, the biggest difference to deformation cloud before and after installing grouted clamp is that the pipe protected by grouted clamp would not be damaged. This also can be drawn that the reinforcement effect of grouted clamp for pipeline is very well.

\section{Mechanical Experiment Analysis of Grouted Clamp}

The pipeline takes axial direction pressure to contrast the buckling pressure before and after installing grouted clamp[13-15]. The ANSYS simulation analysis was done to measure the stress of pipeline in different pressure. The experimental results as showed in Figure 6. 


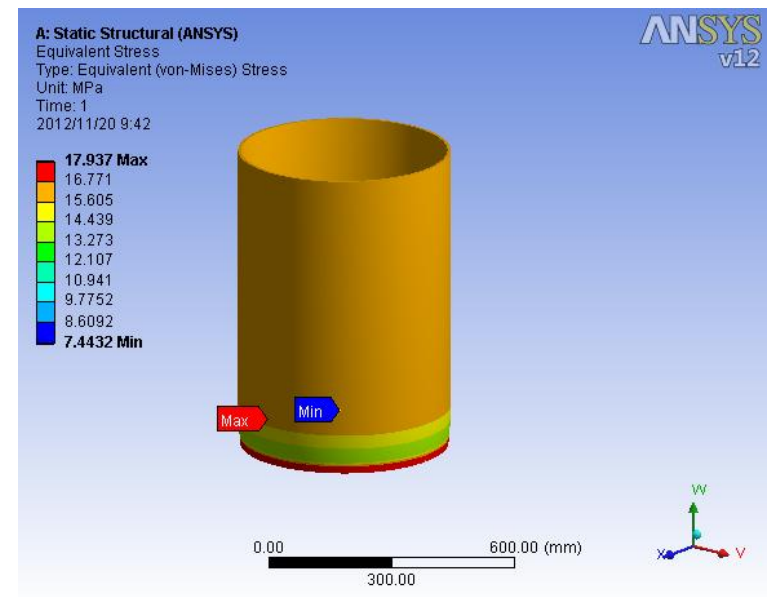

Figure 6. Stress of pipeline in different pressure

As can be seen from the figure, the stress distribution of pipeline is uniform and stress larger phenomenon only appears in the pipe fixed end, therefore the strain gages affixed to the middle position of the pipeline. At the same time as the accuracy of the experiment, four strain gages pastes evenly along the ring in the middle position of the pipeline. Four strains gauge distributed into figure ten. Strain gage is adhered as showed in Figure 7.

Using simulation method to exert pressure on the pipeline and the pressure is constantly increasing. When the pressure reached 50 tons, the maximum stress of pipeline closed to the yield limit of pipeline's material. Therefore, the pressure of 200 tons was selected to experiment. The experimental setup is shown in Figure 8.

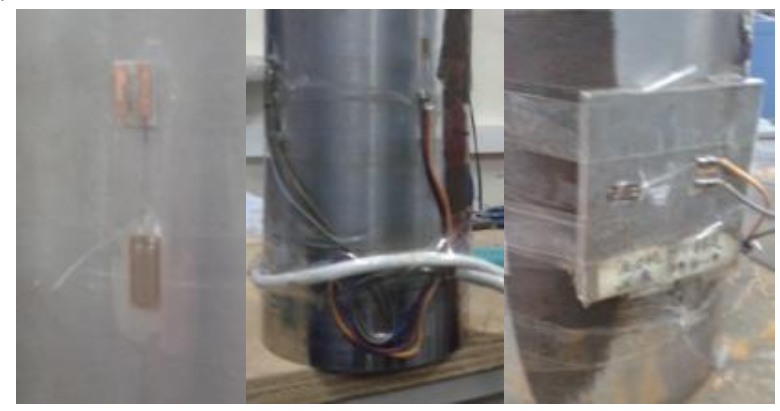

Fig. 7. The distribution of strain gauges

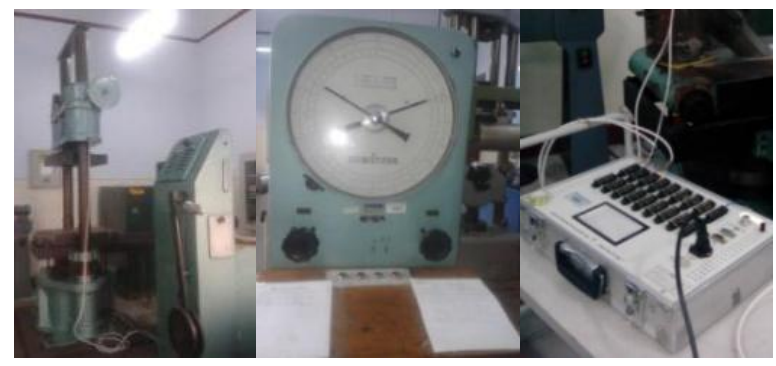

Fig. 8. Test equipment of pressure

When the applied pressure is small, the stress changes little. Therefore the presses applied start from 10 tons until the emergence of yielding phenomenon.

Four strain gages were numbered in accordance with the order in the pressing process and recorded the strain by strain gauges. And then the average value was calculated. We can calculate the stress value according to the stress-strain formula that $\sigma$ is equal to $E$ multiplied by $\varepsilon_{0}$. The material of pipeline is cold rolled steel sheet, so the young's modulus of cold rolled steel is $2.05 \mathrm{E} 11 \mathrm{MPa}$ according to mechanical manual. When the pressure reached $47 \mathrm{MPa}$, the material will yield. The recorded data as showed in Table 3. 
Table 3. The results of mechanical test

\begin{tabular}{|c|c|c|c|c|c|c|}
\hline Force(t) & Strain1 & Strain2 & Strain3 & Strain4 & $\begin{array}{c}\text { Average } \\
\text { strain }\end{array}$ & Stress(MPa) \\
\hline 10 & -495 & -258 & -356 & -254 & -341 & 69.85 \\
\hline 15 & -666 & -424 & -515 & -431 & -509 & 104.34 \\
\hline 20 & -839 & -596 & -674 & -618 & -682 & 139.75 \\
\hline 25 & -971 & -740 & -792 & -765 & -817 & 167.48 \\
\hline 30 & -1169 & -950 & -980 & -1004 & -1026 & 210.27 \\
\hline 35 & -1362 & -1179 & -1158 & -1249 & -1237 & 253.58 \\
\hline 45 & -1574 & -1636 & -1512 & -1780 & -1626 & 333.22 \\
\hline 48 & -1896 & -2024 & -2000 & -2000 & -1980 & 405.90 \\
\hline
\end{tabular}

After completion of straight mechanics experiment, then having mechanics experiment for the pipe which is installed clamp and grouting completed in order to verify the effect of reinforcement and maintenance of the pipeline by the clamp. The strain gauges can't be mounted on the outside of the pipe which has the clamp, then it should be attached to the inside of the pipe, which is shown in Figure 9.

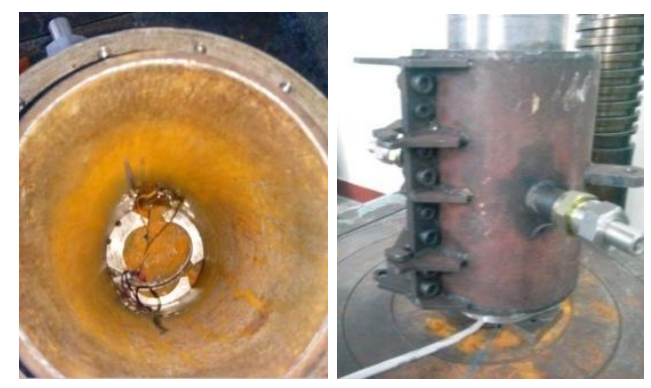

Fig. 9. The distribution of strain gauges

Considering that it is difficult to install the strain gauges on the inside of the pipe, thus, in this experiment, paste only the two strain gauges in the middle of the pipeline with circumferential position of the line direction.

The pressure is applied to the pipeline with clamp and without clamping with the same values in this pressing process in order to verify the effect of reinforcement and maintenance of the pipeline by the clamp. Two strains gauge are numbered and recorded, then calculated the average value $\varepsilon 0$, according to the stress-strain formula $\sigma=E \varepsilon 0$ to stress the value. The material of pipeline is cold-rolled steel sheet; its young's modulus is $2.05 \mathrm{e} 11 \mathrm{MPa}$ according to mechanical manual. When the pressure reached $47 \mathrm{MPa}$, the material will yield. The recorded data are shown in Table 4.

Table 4. The results of mechanical test after installing clamp

\begin{tabular}{|c|c|c|c|c|}
\hline Force(t) & Strain1 & Strain2 & Strain3 & Stress(MPa) \\
\hline 15 & 451 & 371 & 411 & 84.25 \\
\hline 20 & 621 & 515 & 568 & 116.44 \\
\hline 25 & 772 & 664 & 718 & 147.19 \\
\hline 30 & 861 & 785 & 823 & 168.71 \\
\hline 35 & 1046 & 965 & 1006 & 206.12 \\
\hline 40 & 1199 & 1093 & 1146 & 234.93 \\
\hline 45 & 1349 & 1024 & 1187 & 243.23 \\
\hline
\end{tabular}

Mechanics experimental data of pipeline with clamp and without clamping are contrasted and analyzed; the recorded data is shown in Table 5. 
Table 5. The contrast of mechanical test before and after installing clamp

\begin{tabular}{|c|c|c|c|}
\hline Force (T) & Stress of clamp(MPa) & Stress of pipe(MPa) & Difference (MPa) \\
\hline 15 & 84.25 & 104.34 & 20.09 \\
\hline 20 & 116.44 & 147.24 & 30.80 \\
\hline 25 & 133.25 & 167.48 & 34.23 \\
\hline 30 & 168.71 & 210.27 & 41.56 \\
\hline 35 & 206.12 & 253.58 & 47.45 \\
\hline 40 & 258.91 & 299.14 & 40.23 \\
\hline 45 & 243.23 & 333.22 & 90.02 \\
\hline
\end{tabular}

It can be seen from Table 5. Stress of pipeline with clamp is much smaller than the pipeline without clamp at the same stress before the material yield, and the difference increase gradually.

The data in Table 5 were drawn in order to clear the effect of reinforcement and maintenance of the pipeline by the clamp, which is shown in Figure 10.

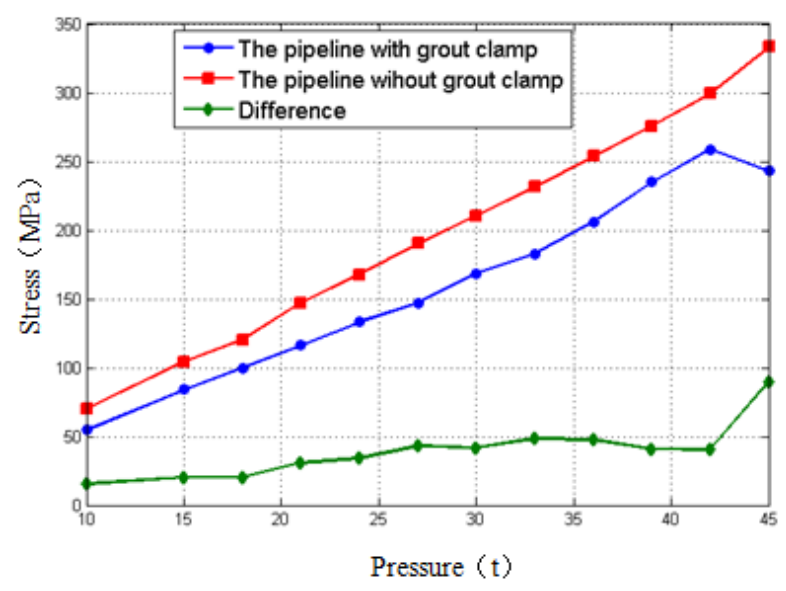

Fig.10. The relation of stress and presure on pipeline

As can be seen from Figure 10, the axial stress of the pipeline with clamp decreased significantly under the same positive stress, which illustrated well the effect of reinforcement and maintenance of the pipeline by the clamp and corresponded with the above theoretical analysis results.

\section{Conclusions}

According to the research objectives of strengthening and reinforcing local topics damaged underwater offshore platform structure, This paper mainly studies the effect of reinforcement and maintenance of the damaged pipeline by the grouting reinforcement clamp device, which analyzed respectively from three aspects of buckling theory analysis, finite element analysis and grouted clamp mechanics experiment, which can prove the feasibility of the effect of reinforcement and maintenance of the pipeline by the clamp and improve the safety and reliability of offshore platforms significantly.

\section{Acknowledgements}

This material is based upon work supported by the National Science Foundation of China (Grant no. 51479043). The views expressed are authors alone.

\section{References}

[1] X.H.Zhan, Ocean University of China,1,(2012) 
[2] H.Y.Cui, J Vibra Shock,27,156(2008)

[3] Y.L, L.X, H.F.Deng, J. Saf. Sci.Tech,9,119(2013)

[4] S.F.Gong, W.L.Jin,Q.Z.Wang, CHN.O.P,16,38(2001)

[5] J.B.Zhang, Jin, Q.Z.Wang, CHN.O.P,19,45(2004)

[6] S.F.Gong, X.W.Shen, F.Li, Ocean Eng,19,32(2001)

[7] W.L.Jin, J.Song, S.F.Gong, Eng. Mech, 20,100(2003)

[8] X.Shi, X.H.Zhan, B.Yang, Ocean Eng,29,77(2011)

[9] X.Shi, X.H.Zhan, B.Yang, Ocean Eng,31,79(20131)

[10] Z.Wang, S.C.Jiang, J.Zhang, Prog. S. Bui. Stru,12, 11 (2010)

[11] X.G.Wu, J. Harbin Eng.Uni,30,635(2009)

[12] N.S.Wang, Zhejiang University.34(2013)

[13] American Petroleum Institute. API RP2A-WSD.356 (2000)

[14] R.L.Reed, J.K.Smith, OTC, 6652(2001)

[15] MSL Engineering Ltd, MSL Doc., Ref. C357R001,1,83(2004) 